\title{
Precision grid and hand motion for accurate needle insertion in brachytherapy
}

\author{
Carl S. McGill a) and Jonathon A. Schwartz \\ Biomedical Engineering Department, University of Michigan, Ann Arbor, Michigan 48109 \\ Jason Z. Moore \\ Mechanical Engineering Department, University of Michigan, Ann Arbor, Michigan 48109
}

Patrick W. McLaughlin

Department of Radiation Oncology, University of Michigan, Ann Arbor, Michigan 48109

Albert J. Shih

Biomedical Engineering and Mechanical Engineering Departments, University of Michigan, Ann Arbor, Michigan 48109

(Received 16 February 2011; revised 19 June 2011; accepted for publication 20 June 2011; published 29 July 2011)

\begin{abstract}
Purpose: In prostate brachytherapy, a grid is used to guide a needle tip toward a preplanned location within the tissue. During insertion, the needle deflects en route resulting in target misplacement. In this paper, 18-gauge needle insertion experiments into phantom were performed to test effects of three parameters, which include the clearance between the grid hole and needle, the thickness of the grid, and the needle insertion speed. Measurement apparatus that consisted of two datum surfaces and digital depth gauge was developed to quantify needle deflections.

Methods: The gauge repeatability and reproducibility (GR\&R) test was performed on the measurement apparatus, and it proved to be capable of measuring a $2 \mathrm{~mm}$ tolerance from the target. Replicated experiments were performed on a $2^{3}$ factorial design (three parameters at two levels) and analysis included averages and standard deviation along with an analysis of variance (ANOVA) to find significant single and two-way interaction factors.

Results: Results showed that grid with tight clearance hole and slow needle speed increased precision and accuracy of needle insertion. The tight grid was vital to enhance precision and accuracy of needle insertion for both slow and fast insertion speed; additionally, at slow speed the tight, thick grid improved needle precision and accuracy.

Conclusions: In summary, the tight grid is important, regardless of speed. The grid design, which shows the capability to reduce the needle deflection in brachytherapy procedures, can potentially be implemented in the brachytherapy procedure. (C) 2011 American Association of Physicists in Medicine. [DOI: 10.1118/1.3611040]
\end{abstract}

Key words: prostate brachytherapy, needle grid, needle deflection

\section{INTRODUCTION}

Position accuracy of the needle tip to place the radioactive seeds into the prostate is important for optimizing the dose delivery and efficacy to cancerous tissue. This treatment, called brachytherapy, is performed to eradicate cancerous cells within the prostate gland. Inaccurately, placed seeds can result in adverse side effects such as urinary and bowel incontinence, rectal bleeding, erectile dysfunction, and substantial tissue damage. ${ }^{1-3}$

Accurate seed placement is determined by the placement of the needle tip in the tissue. Transperineal image-guided brachytherapy enables real-time localization of needle positioning. ${ }^{3,4}$ A major problem experienced with this brachytherapy procedure is that the needle deflects en route to the target due to the inherent tissue deformation caused by the soft and heterogeneous tissue properties, the unfavorable anatomic structures in and around the prostate, and the limited maneuverability with needle insertion. ${ }^{1-3}$
To improve the needle insertion accuracy in brachytherapy, investigators have studied the needle insertion process and prostate deformation in an attempt to model the needle-tissue interaction, ${ }^{5-7}$ to predict the needle deflection and the needle path based on measured needle insertion forces, ${ }^{8-13}$ and to improve the needle insertion accuracy through the use of a robotic-assisted tool. ${ }^{9,14-16}$ Abolhassani et al. ${ }^{17}$ have an in-depth survey of the needle trajectories, tissue deformation modeling, and various other needle-tissue interaction properties during the needle insertion. To our knowledge no studies have been conducted on the effect of the grid, which supports the needle during insertion, and speed of needle generated by hand motion and its outcome on the accuracy of needle placement.

In brachytherapy, the orientation of the needle during insertion is constrained by a grid, also referred to as a template. $^{3,4}$ A grid, ${ }^{18,19}$ as shown in Fig. 1(a), consists of an 
array of equally spaced holes, which have the inside diameter closely matching the outside diameter of the needle. The 18 -gauge needle, having a $1.270 \pm 0.013 \mathrm{~mm}$ outer diameter, is commonly used during brachytherapy. The needle is guided by a hole in the grid into a preplanned target location within the soft tissue. The needle grid (Civco Medical Solutions, IA), as shown in Fig. 1(a), is made of plastic and has a $13 \times 13$ array of holes. The spacing between adjacent holes is $5 \mathrm{~mm}$ and the thickness of the grid is $20.3 \mathrm{~mm}$. The diametral clearance between the outside diameter of the needle and hole inside diameter of the current commercially available 18-gauge grid is about $76 \mu \mathrm{m}$, which is large from the precision engineering perspective. This study applies a precision reaming process to produce a stainless steel grid that has smaller and more consistent hole size for needle guidance. The diametral clearance between the new grid inside diameter hole and the outside diameter needle is reduced to $33 \mu \mathrm{m}$. One of the goals of this study is to investigate the effect of the reduction of this clearance on the accuracy of needle insertion.
In current brachytherapy procedures, physicians in radiation oncology manually insert the needle through the grid and into the prostate gland, with a quick flick of the wrist to achieve a high-needle speed and less deflection of needle. Another goal is to study the effect of this hand motion in needle insertion on the accuracy of needle placement.

Quantifying needle deflection provides a means to compare and evaluate improvements on accuracy. Techniques used to measure deflection or track the needle path include reconstructing the stereo images of a biplane x-ray system, ${ }^{10}$ inserting inside the needle a five-degrees of freedom sensor coil that uses a magnetic field as part of the setup, ${ }^{11,20}$ and placing graph papers on each ends of the phantom. ${ }^{21,22}$ These methods do not have documented measurement accuracy. The wellestablished gauge repeatability and reproducibility (GR\&R) (Refs. 23-25) measurement test in precision metrology can quantify the error of these measurement techniques for needle deflection. The final goal of this study is to design a measurement system that has acceptable GR\&R results and documented accuracy on needle deflection measurements.
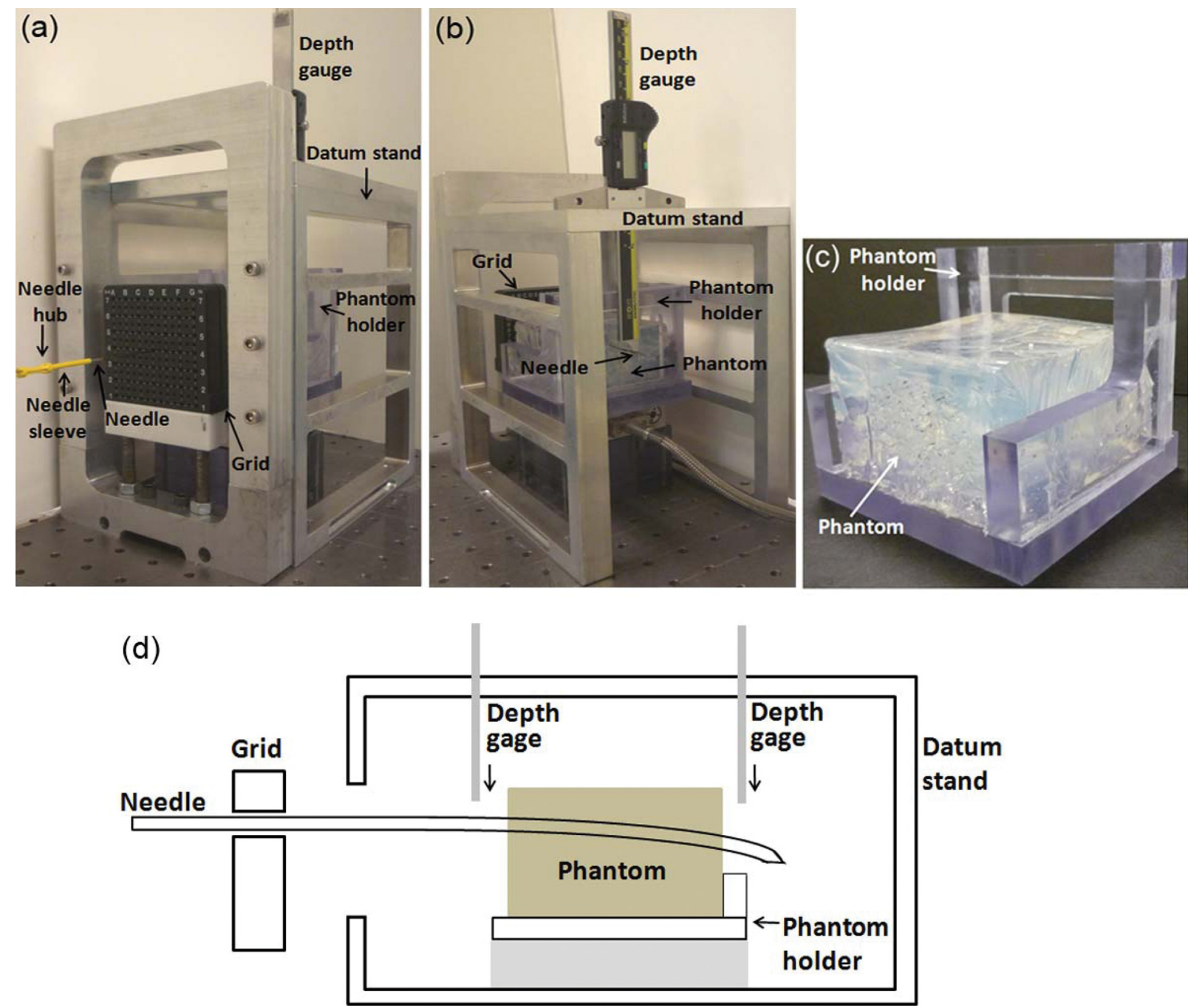

FIG. 1. (a) Front-view and (b) rear-view of the experimental setup which includes the grid, needle, phantom, phantom holder, measurement datum stand, and depth gauge. (c) Phantom holder and phantom with an opening on the back of the phantom holder that allows the needle to exit. (d) Schematic drawing of experimental setup. 


\section{MATERIALS AND METHOD}

\section{II.A. Overview of experimental setup}

The setup (Fig. 1) for the experiment includes a needle, phantom, phantom holder, grid, measurement datum stand, and digital depth gauge. The needle used throughout the experiment was the commonly used 18-gauge prostate brachytherapy needles (Model MTP-1820-C, Mick RadioNuclear Instruments Inc, NY). The brachytherapy needle consists of two main parts, the inner solid trocar and outside hollow cannula, both made of AISI 304 stainless steel. The trocar, which is the inner solid rod that converges to a diamond point (3-plane) at one tip, has a diameter of about $1.01 \mathrm{~mm}$. Enclosing the trocar is the hollow cannula. At the opposite end of the diamond tip of the trocar is a yellow covering, called the hub [Fig. 1(a)]; likewise, there is also a yellow covering on the cannula opposite the tip, called the needle sleeve. The hub and needle sleeve provide a means to hold and insert the needle combination during the brachytherapy procedure. Bevel tip needles were not considered because diamond tip needles, commonly used in brachytherapy, produced less deflection when inserted into similar homogenous phantom, as shown by Podder et al. ${ }^{21}$

The phantom was made of polyvinylchloride (PVC) modified with plastisol, previously used by Podder et al., ${ }^{21,26}$ and was created from a 1:1 ratio of regular liquid plastic to plastic softener (M-F Manufacturing, TX). In order to have a consistent phantom specimen throughout the entire experiment, one batch of mixture was produced and cut into two separate phantom blocks with the stated dimensions. One phantom block was formed to have a length of $85 \mathrm{~mm}$ that the needle traveled through; this length was also used to measure needle deflection within the phantom. A width of $75 \mathrm{~mm}$ was molded to accommodate the phantom height of $50 \mathrm{~mm}$. This width-height aspect ratio provided enough self-support for the phantom to stay on the phantom holder as well as enough space for needle insertion at several height levels of holes in the grid.

The phantom holder [Fig. 1(c)], $90 \mathrm{~mm}$ long $\times 100 \mathrm{~mm}$ wide $\times 75 \mathrm{~mm}$ high, was made from $12.7 \mathrm{~mm}$ thick polycarbonate and used to secure the phantom and provides a constant location to measure the needle for position coordinates. The phantom holder encloses some of the phantom on the right and left sides and the entire phantom on the bottom. An opening on the back of the phantom holder allows the needle to exit the phantom during needle insertion. A constant distance of $25 \pm 5 \mathrm{~mm}$ was set between the phantom and the front of each interchangeable grid for every needle insertion trial. This distance is a representative value of the gap between the patent's skin and the grid, which is present during brachytherapy procedures.

The next section refers to Secs. II A 1 Grids and II A 2 Needle deflection measurement apparatus.

\section{II.A.1. Grids}

The two factors of interest related to the grid were the tightness of the hole and the length of the grids. Four sets of grids, as shown in Fig. 2, were used in the experiment. One is the plastic grid (Civco Medical Solutions, IA), as shown in Fig. 2(a), used in current brachytherapy procedures for 18 -gauge needles. This grid, marked as the loose thin grid in Fig. 2(a) and Table I, has a $13 \times 13$ array of equally spaced $1.346 \mathrm{~mm}$ diameter holes and a thickness of $20.3 \mathrm{~mm}$. Two plastic grids were stacked together to increase the thickness to $40.6 \mathrm{~mm}$, which is marked as loose thick in Table I and shown in Fig. 2(b).

Metal grids with a $13 \times 13$ array of equally spaced holes, the same configuration as the current plastic grid, were constructed. To determine the hole size of the metal grid for tighter diametral clearance, a set of holes with 6 $\mu \mathrm{m}$ size step was constructed for testing and selecting the suitable hole size. A radiation oncologist performed test insertions on this grid to feel and find the smallest hole that had the same level of resistance on the needle as the resistance encountered in the operating room, when the needle is inserted for the brachytherapy procedure. The $1.303 \mathrm{~mm}$ hole diameter, marked as tight grids in Fig. 2 and Table I, was selected. The diametral clearance between the $1.303 \mathrm{~mm}$ hole and the 18-gauge needle is about $33 \mu \mathrm{m}$.

The reaming tool to produce the precision hole has limited length and can only produce $12.7 \mathrm{~mm}$ deep hole. In this study, as shown in Figs. 2(c) and 2(d) and listed in Table I, the tight thin and tight thick grids are the stacking of two and four of these precise holes $12.7 \mathrm{~mm}$ thick metal plates producing 25.4 and $50.8 \mathrm{~mm}$ thickness, respectively, using precision dowel pins and screws.

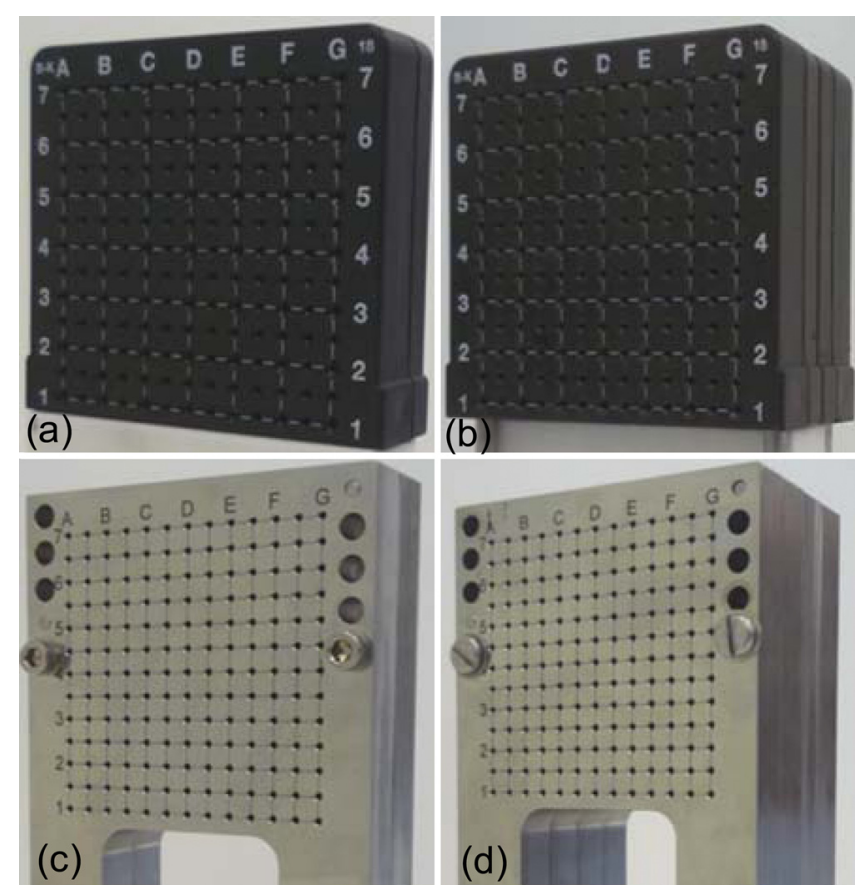

FIG. 2. Grids-isometric view of the four (a) loose thin, (b) loose thick, (c) tight thin, and (d) tight thick grids in this study. (a) and (b) are the plastic grids with (c) and (d) are the 316 stainless steel metal grids. 
TABLE I. Specification of the four needle grids.

\begin{tabular}{lcc}
\hline \hline Grid type & Grid thickness $(\mathrm{mm})$ & Hole diameter $(\mathrm{mm})$ \\
\hline Loose thin & 20.3 & 1.346 \\
Loose thick & 40.6 & 1.346 \\
Tight thin & 25.4 & 1.303 \\
Tight thick & 50.8 & 1.303 \\
\hline \hline
\end{tabular}

\section{II.A.2. Needle deflection measurement apparatus}

The measurement apparatus, as shown in Figs. 1(a) and 1(b), includes the datum stand, attached to the grid holder, and a digital depth gauge (Mitutoyo Model VDS-6 DCX) with $0.01 \mathrm{~mm}$ resolution. The same depth gauge was used to mechanically measure the needle position coordinates in the $x$ - and $y$-directions from the $x$ - and $y$-datum surfaces, as shown in Fig. 3. The $x$ - and $y$-coordinates of the needle after it enters and exits the phantom are marked as $\left(X_{\text {enter }}, Y_{\text {enter }}\right)$ and $\left(X_{\text {exit }}, Y_{\text {exit }}\right)$, respectively, and measured using the same depth gauge, as illustrated in Fig. 3. The depth gauge base was parallel to the respective datum plane. The needle deflections through the $85 \mathrm{~mm}$ phantom length are denoted as $X_{\text {deflection }}$ and $Y_{\text {deflection }}$ and can be calculated using

$$
\begin{aligned}
& X_{\text {deflection }}=X_{\text {enter }}-X_{\text {exit }}, \\
& Y_{\text {deflection }}=Y_{\text {enter }}-Y_{\text {exit }} .
\end{aligned}
$$

The resultant/radial deflection $(R)$ is

$$
R=\sqrt{X_{\text {deflection }}^{2}+Y_{\text {deflection }}^{2}} \text {. }
$$

\section{II.A.3. Needle insertion speed and phantom deformation}

During the experiment, a needle was inserted by hand at a slow or fast speed through a hole on the grid and into the phantom. The fast speed is created by a quick flick of the wrist commonly used by radiation oncologists in the current hand placed needle insertion brachytherapy procedure. The operator's hand was placed behind the hand plate to push the hand plate and drive the needle to insert through the grid and into the phantom under the guidance of the hand plate. A linear optical encoder (Heidenhain Model Lida 277) was attached to the hand plate to measure the position of the needle during hand insertion, as shown in Fig. 4. The data were recorded using a data acquisition system (National Instruments Model NI DAQPad-6015) and LABVIEW at a sampling rate of $50 \mathrm{kHz}$. The needle insertion speed was calculated based on the displacement and time data. The slow speed was $60 \pm 40 \mathrm{~mm} / \mathrm{s}$, while the fast speed was $525 \pm 60 \mathrm{~mm} / \mathrm{s}$ for the loose thin, loose thick, and tight thin grids and $750 \pm 60 \mathrm{~mm} / \mathrm{s}$ for the tight thick grid. Once inserted, the needle was measured for $x$ - and $y$-coordinates at the entry and exit of the phantom to calculate the deflection. Measurements were repeated for each insertion. Phantom deformation at slow and fast needle insertion speeds was recorded using video.

\section{II.B. Procedure for Gauge Repeatability and Reproducibility (GR\&R)}

GR\&R was performed on the datum stand by the procedure outlined by Stamatis. ${ }^{24}$ This procedure requires ten trials, the same three operators measuring each trial, and each operator measuring each trial three times, with each trial consisting of four position measurements $\left(X_{\text {enter }}, X_{\text {exit }}, Y_{\text {enter }}\right.$, and $Y_{\text {exit }}$. For the GR\&R, an operator performed the test as follows: one needle was inserted through the grid, very slowly by hand and into the phantom. The operator obtained position coordinates of the needle in the order of $X_{\text {enter }}$ and $X_{\text {exit }}$ for the $x$-direction and $Y_{\text {enter }}$ and $Y_{\text {exit }}$ for the $y$-direction and recorded the values for Measurement \#1 data. After
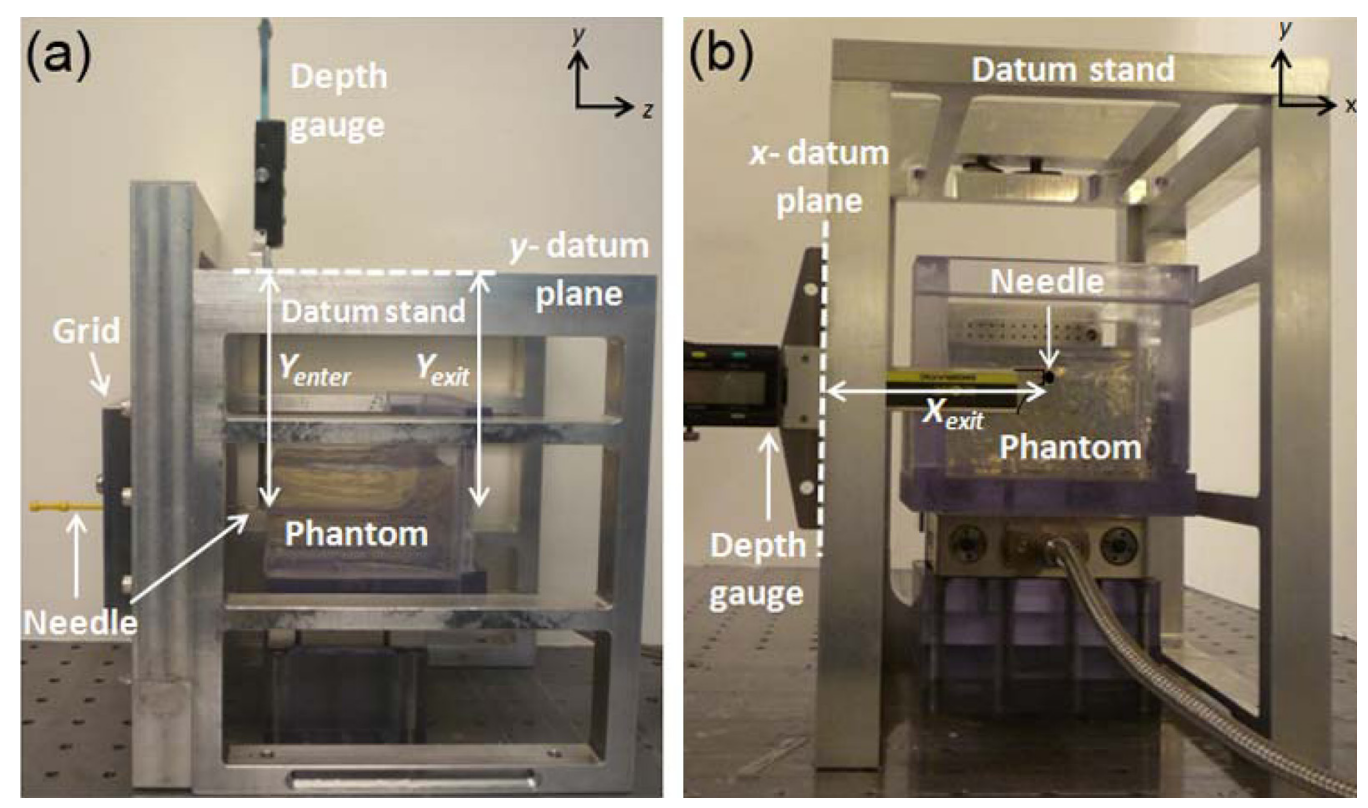

FIG. 3. Needle deflection measurement apparatus with the datum stand, $x$-and $y$-datum planes, and precise digital depth gauge to measure the needle positions (a) side view of the needle, grid, depth gauge, $y$ - datum plane and measured $Y_{\text {enter }}$ and $Y_{\text {exit }}$ and (b) back view of $x$ - datum plane and measured $X_{\text {enter }}$ and $X_{\text {exit }}$. 


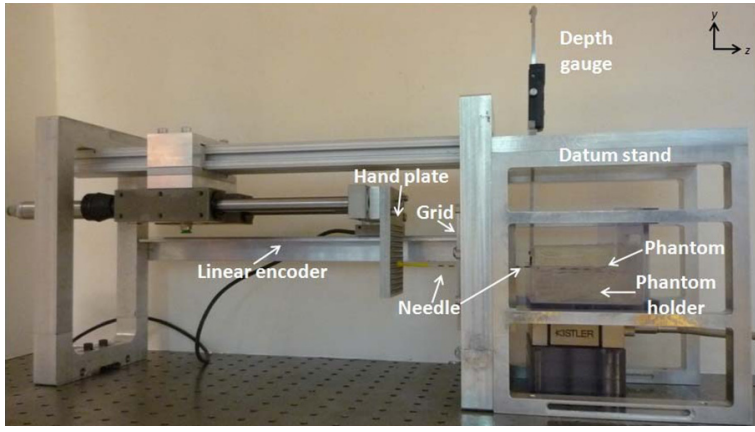

FIG. 4. Experimental setup which displays the linear encoder and hand plate used to measure the position of the needle during hand insertion. Needle insertion speed was calculated from displacement and time data.

completing and documenting the data on the first set of measurements, the operator remeasured the needle for position coordinates two more times. Three operators independently executed the measurements on the same inserted needle, performing the same measurement procedure as outlined. After the first needle insertion was performed, nine more insertion trials using a different hole location in the grid were made, for a total of ten needle insertion trials, and similar measurements were performed on the needle by the three operators. Analysis to the data was performed as outlined by Stamatis. ${ }^{24}$

\section{II.C. Design of Experiments}

\section{II.C.1. Factors and conditions}

A $2^{k}$ factorial design experiment, ${ }^{27}$ where $k=3$ (factors) at two levels or values, was performed in this study. In a factorial design, all possible combinations of factor levels are tested. As shown in Table II, the three factors were needle speed, grid thickness, and grid tightness. The $2^{3}$ factorial design experiment produced eight condition combinations, each having ten replicates for a total of 80 data points for the entire experiment. A sample size of $n=10$ was obtained using an operating characteristic (OC) curve and appropriate sample size equation, ${ }^{27}$ discussed in Appendix . Because of the extensive time required to measure the needle position coordinates along with randomizing the order of the replicates and grids, the experiment was performed over two days, with four conditions executed in one day. In Table II, the first column identifies the eight conditions used in experimentation, while the next three columns state the factor level at the respective condition. The last few columns show which conditions are studied: main effects (the overall effect of one factor), two-way interaction (the effect of one factor depending on the level of the second factor-total of two factors simultaneously), and three-way interaction (the effect of two factors depending on the level of the third factortotal of three factors simultaneously). Conditions shown in Table II were arranged so that the three main effects and the three-way interaction, between factors were performed on day two, while the other four conditions would be performed on day one.

\section{II.C.2. Grid coordinates and alignment}

Pilot studies indicated that the starting location of the needle on the grid can influence needle deflection due to deformation of the phantom. In statistics, a Latin square design ${ }^{27}$ is a $p \times p$ square array containing $p$ rows and $p$ columns and is used to eliminate sources of variability from the row and column factors. To account for any grid effects on the data, a Latin square design was adapted into a "Latin rectangle" because of different number of rows versus columns. This Latin rectangle will enable grid hole locations to distribute effects equally among the condition combinations. As shown in Fig. 5 , a $5 \times 8$ region in the lower central region of the grid was selected to conduct the needle insertions. The region was selected so that repeatable results of each trial could be achieved, as shown in the preliminary tests. The number scheme was developed to confirm that all condition combinations were performed at each row number and column letter; each grid coordinate used only once per day, for each needle insertion. One set of roman numeral represents the ten coordinates needed for the ten replicates for each conditional combination, i.e., all I's in the grid holes are for test \#6 (on day one) and test \#3 (on day two). The region in Fig. 5 presents four sets of ten holes to use on one phantom when performing the four condition combinations experiment on one day.

Two phantom blocks, one block per day, were used in this experiment. The phantom block was kept in the same position within the phantom holder and behind the grid. With only one needle insertion occurring per grid coordinate,

TABLE II. Design matrix of condition combinations including day assignment.

\begin{tabular}{|c|c|c|c|c|c|c|c|}
\hline \multirow[b]{2}{*}{ Test \#: Conditions studied } & \multicolumn{3}{|c|}{ Factors } & \multirow[b]{2}{*}{ Main effect } & \multicolumn{2}{|c|}{ Interactions } & \multirow[b]{2}{*}{ Day } \\
\hline & Needle Speed & Grid Thickness & Grid Tightness & & Two-way & Three- way & \\
\hline 1: Control & slow & thin & loose & - & - & - & 1 \\
\hline 2: Speed & fast & thin & loose & $\mathrm{X}$ & - & - & 2 \\
\hline 3: Thickness & slow & thick & loose & $\mathrm{X}$ & - & - & 2 \\
\hline 4: Speed/thickness & fast & thick & loose & - & $\mathrm{X}$ & - & 1 \\
\hline 5: Tightness & slow & thin & tight & $\mathrm{X}$ & - & - & 2 \\
\hline 6: Speed/tightness & fast & thin & tight & - & $\mathrm{X}$ & - & 1 \\
\hline 7: Thickness/tightness & slow & thick & tight & - & $\mathrm{X}$ & - & 1 \\
\hline 8: Speed/thickness/tightness & fast & thick & tight & - & - & $\mathrm{X}$ & 2 \\
\hline
\end{tabular}




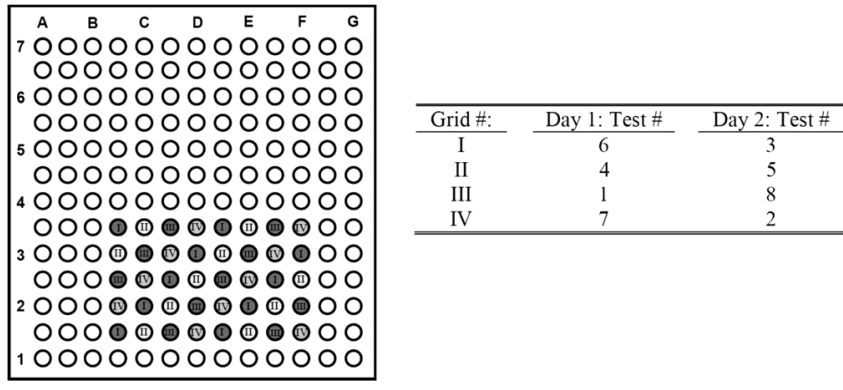

FIG. 5. Region of the grid chosen for experiment to distribute effects equally among condition combinations. Roman numerals in the grid correspond to a certain test number, depending on which day the test was performed, as shown in the legend.

every needle entered a new area within the phantom. Furthermore, with two adjacent grid holes having $5 \mathrm{~mm}$ distance of separation, a needle was always inserted into an unused area within a phantom. As shown by the $5 \times 8$ grid coordinates (a total of 40 holes) in Fig. 5, one insertion occurred into each hole within the grid and phantom, for a total of 80 insertion holes within two phantom blocks over the two day experiment. This experimental design eliminates the mechanical damage created from inserting multiple needles within the same grid coordinates and same needle area of the phantom, thus not affecting needle deflection results later in the experiment.

Preceding each needle insertion, the grid was aligned to the two datum surfaces on the datum stand via measuring a reference needle, which was placed through a hole that does not insert into the phantom and adjusting the orientation of the grid to produce a zero deflection on the needle.

\section{II.C.3. Full randomization}

Randomization was used for the following: (1) Assignment of the factors to the coding letters A, B, and C used for analysis purposes; (2) Day assignment of the four condition combinations; (e.g. main effects and three-way interaction performed together either on day one or day two) (3) Assignment of the group of ten coordinates to a condition combination that was used for the ten replicates; and (4) the order of the grid coordinates/replicates with the respective grid within the day assignment. Furthermore, a full randomization within each day along with the entire experiment occurred.

\section{II.D. Analysis methods}

Analyses were preformed on the $X_{\text {deflection }}, Y_{\text {deflection, }}$, and $R$ data separately. Standard deviation of measured data was used to evaluate the precision and closeness of the replicates to each other within a grid type. Averages of the needle deflection data provided an approach to compare accuracy within a grid type. Furthermore, deflection effects were used to analyze the data to provide information on whether any factor(s) had significant effect ( $p$-value $<0.05$ ) on needle deflection. The response variables were all 80 data points collected during the experiment. The $X_{\text {deflection }}, Y_{\text {deflection, }}$ and $R$ data were entered as the response variables into the statistical software, MINITAB (State College, PA), while speed, thickness, and tightness were used as the two-level factors. A $2^{3}$ factorial design analysis of variance (ANOVA) was performed on the $X_{\text {deflection }}, Y_{\text {deflection, and } R \text { data; coded }}$ units, -1 for low settings (e.g., all three factor levels for test \#1) and + 1 for high settings (e.g., "fast" needle speed for test \#2), was used to define the factor levels. Coded units enable the experimental design to be orthogonal, which allows MINITAB to estimate model terms independently. Main effects and two-way interactions were of interest for significant factors. After identifying significant factors, Tukey's test ${ }^{27}$ was performed on the highest order terms (significant two-way interactions) in the model to find which means were significantly different from one another.

\section{RESULTS AND DISCUSSIONS}

\section{III.A. GR\&R Results}

Results in Table III demonstrated that the measurement apparatus developed in this study (Figs. 1 and 3) is capable to measure the $2 \mathrm{~mm}$ needle deflection. GR\&R equations utilize a tolerance value in order to calculate the product tolerance used by repeatability error (RPT) within an operator and product tolerance used by reproducibility error (RPD) between operators. Using a tolerance of $2 \mathrm{~mm}$ from the target, the RPT, RPD, and GR\&R for $X_{\text {enter }}, X_{\text {exit }}, Y_{\text {enter }}$, and $Y_{\text {exit }}$ were computed, as shown in Table III. The $2 \mathrm{~mm}$ tolerance was obtained from the radiation oncologist who performs brachytherapy procedures regularly. As shown in Table III, all values of the GR\&R were less than $30 \%$. Shina $^{25}$ described GR\&R as the total variation (due to repeatability and reproducibility error) used to determine if the measurement system is acceptable for its intended applications. A GR\&R less that $10 \%$ is acceptable; between $10 \%$ to $30 \%$ it is marginally acceptable; greater than $30 \%$ the GR\&R is not acceptable. ${ }^{25}$ If the tolerance is reduced to 1 $\mathrm{mm}$, the current measurement apparatus will not be capable for the measurement because the GR\&R for $X_{\text {enter }}$ and $Y_{\text {exit }}$ are both larger than $15 \%$. Furthermore, a $2 \mathrm{~mm}$ tolerance is acceptable for measuring needle deflection.

\section{III.B. Average and standard deviation of needle deflection}

Using Grubbs' test, ${ }^{28,29}$ discussed in Appendix, one of the replicates from the tight thin grid with fast insertion was tested as an outlier. This replicate's $Z$ value was 2.33 , greater than the critical values of 2.29 (for $n=10$ ), thus that data point was removed from the study. The measured $X_{\text {deflection }}$ and $Y_{\text {deflection }}$ for the remaining 79 tests are plotted in Fig. 6.

TABLE III. GR\&R results for $X_{\text {enter }}, X_{\text {exit }}, Y_{\text {enter }}$, and $Y_{\text {exit. }}$

\begin{tabular}{ccccc}
\hline \hline Error & $X_{\text {enter }}$ & $X_{\text {exit }}$ & $Y_{\text {enter }}$ & $Y_{\text {exit }}$ \\
\hline RPT (\%) & 12.5 & 10.5 & 6.1 & 12.8 \\
RPD (\%) & 3.9 & 1.2 & 2.9 & 2.3 \\
GR\&R (\%) & 16.4 & 11.7 & 9.0 & 15.1 \\
\hline \hline
\end{tabular}


Analysis was performed on 79 data points for $X_{\text {deflection }}, Y_{\text {de- }}$ flection, and $R$ to obtain the average and standard deviation of the needle deflection, and the results are summarized in Table IV. The average represents the accuracy of the needle insertion, while the standard deviation represents the precision.

Initial observation of Fig. 6 shows large upward $Y_{\text {deflection }}$ data when compared to the $X_{\text {deflection }}$ data. The upward shift in $Y_{\text {deflection }}$ could be explained by the dynamics of the phan-

(a)

$$
\text { Loose Thin }
$$

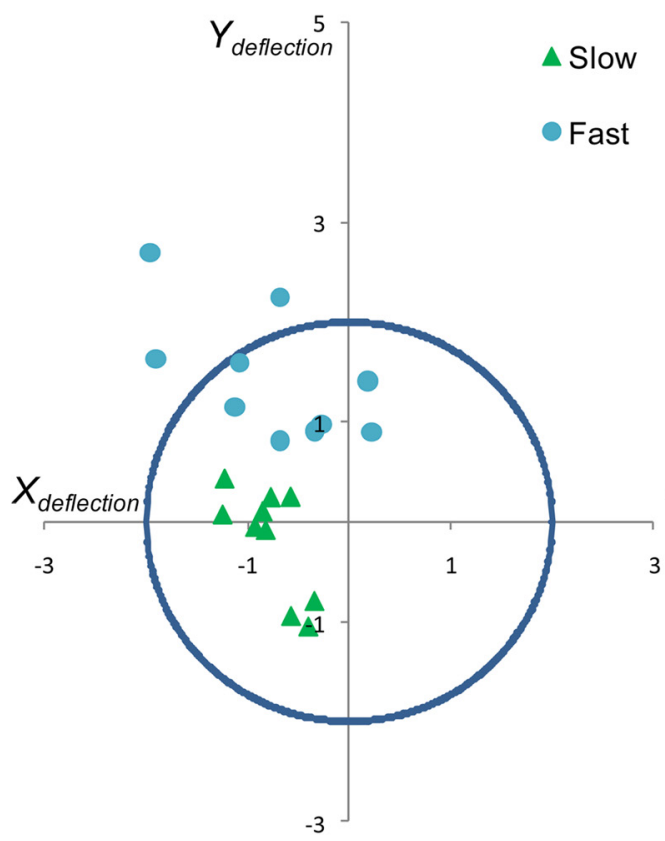

(c)

$$
\text { Tight Thin }
$$

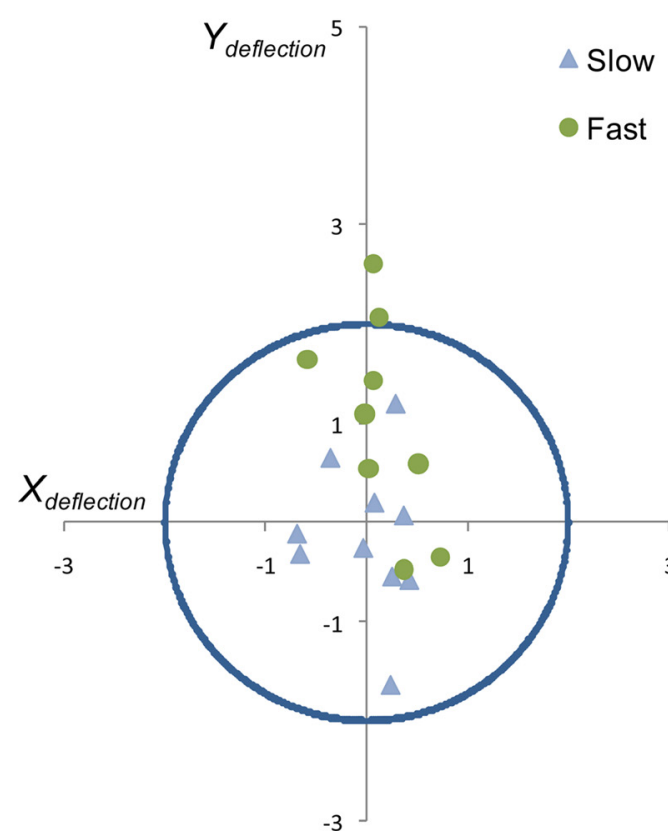

tom during fast insertions. The top surface of the phantom was left unsecure to avoid placing any force on the phantom, thus as the needle inserts into the phantom the top of the phantom bends backward. As the needle moves through the phantom, the phantom readjusts to its original position (the top returning forward), causing the needle to increase in an upward $Y_{\text {deflection, }}$ as shown in Fig. 7. This phenomenon might have led to higher $Y_{\text {deflection }}$ values. However, the Latin

\section{Loose Thick}

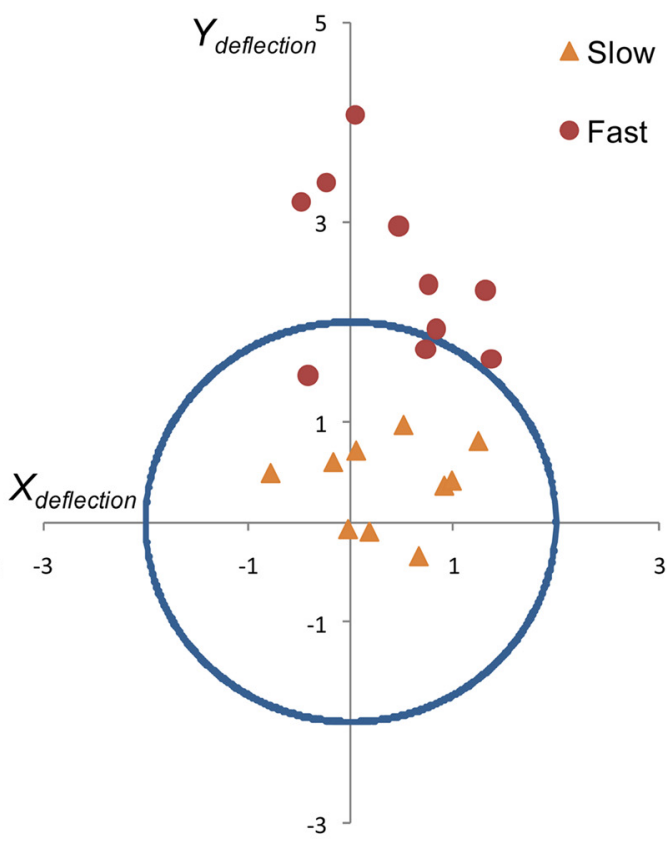

(d)

Tight Thick

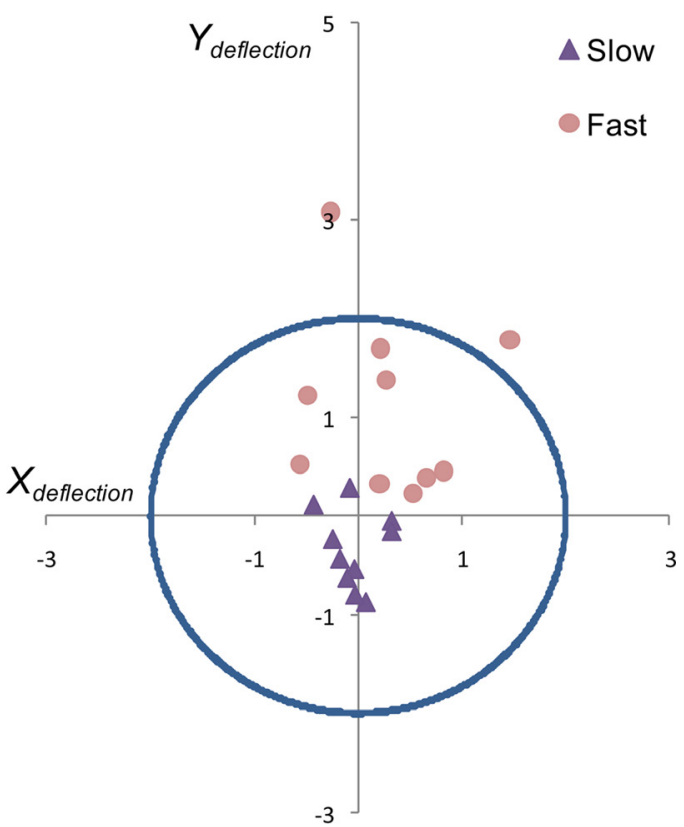

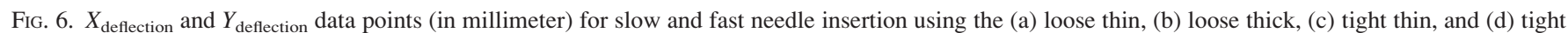
thick grids. A $2 \mathrm{~mm}$ circle is drawn in order to have a means of visualizing how many and how far data points were from target $(0,0)$. 
TABLE IV. Average and standard deviation ${ }^{\mathrm{a}}$ of needle deflection for all four grids.

\begin{tabular}{|c|c|c|c|c|c|c|c|}
\hline \multirow[b]{2}{*}{ Speed } & \multirow[b]{2}{*}{ Grid } & \multicolumn{3}{|c|}{ Average } & \multicolumn{3}{|c|}{ Standard deviation } \\
\hline & & $X_{\text {deflection }}(\mathrm{mm})$ & $Y_{\text {deflection }}(\mathrm{mm})$ & $R(\mathrm{~mm})$ & $X_{\text {deflection }}(\mathrm{mm})$ & $Y_{\text {deflection }}(\mathrm{mm})$ & $R(\mathrm{~mm})$ \\
\hline \multirow[t]{4}{*}{ Slow } & Loose thin & -0.77 & -0.18 & 0.97 & 0.31 & 0.54 & 0.21 \\
\hline & Loose thick & 0.36 & 0.38 & 0.79 & 0.62 & 0.43 & 0.42 \\
\hline & Tight thin & -0.01 & -0.14 & 0.72 & 0.42 & 0.77 & 0.44 \\
\hline & Tight thick & -0.04 & -0.33 & 0.51 & 0.24 & 0.39 & 0.20 \\
\hline \multirow[t]{4}{*}{ Fast } & Loose thin & -0.76 & 1.43 & 1.71 & 0.76 & 0.63 & 0.81 \\
\hline & Loose thick & 0.44 & 2.51 & 2.65 & 0.68 & 0.87 & 0.78 \\
\hline & Tight thin & 0.14 & 1.02 & 1.30 & 0.37 & 1.04 & 0.72 \\
\hline & Tight thick & 0.29 & 1.11 & 1.32 & 0.62 & 0.91 & 0.85 \\
\hline
\end{tabular}

${ }^{\mathrm{a}} 0.01 \mathrm{~mm}$ resolution for measurement tool.

rectangle design of experiment enables all condition combinations to be tested at each row number and column letter within the grid, thus an equal distribution of needle insertion into the grid coordinates occurred for all condition combinations.

In Table IV, average of the needle deflection provides accuracy of the insertion while standard deviation provides precision and closeness of the replicates to each other. The effect of the grid tightness, the grid thickness, and the needle speed are discussed:

(i) Tightness: In all 12 cases for $X_{\text {deflection }}, Y_{\text {deflection, and }}$ $R$ in Table IV, tight grids decrease the average of the needle deflection, when comparison occurred between grids of the same thickness and speed. As far as standard deviation, a tight grid does not necessarily decrease standard deviation. At fast insertion speed, the needle is hard to control during insertion, thus an increase in variation is observed. At slow insertion speed, the tight thick grid has the smallest standard deviation for the $X_{\text {deflection, }}, Y_{\text {deflection, }}$, and $R$ data.

(ii) Thickness: Thickness alone does not have a dominant effect on the average and standard deviation of needle deflection. Our current experimental setup does not improve the average and standard deviation of the needle deflection when a thicker grid was used.

(iii) Speed: Inserting the needle at slow speed reduces both the averages and standard deviation of the needle deflection for most conditions. Only two exceptions for the $X_{\text {deflection }}$ data - the loose thin grid for average needle deflection and the tight thin grid for standard deviation-are observed having a slightly higher $X_{\text {de- }}$ flection value when inserting the needle at slow speed. The $Y_{\text {deflection }}$ and $R$ data for all 16 cases (eight cases for average and eight cases for standard deviation) are consistently lower with slow needle insertion speed.
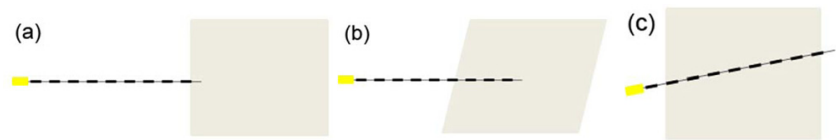

FIG. 7. Large upward $Y_{\text {deflection }}$ data occurs after (a) needle enters phantom, (b) top of phantom deforms as needle penetrates, then (c) phantom undeforms and pushes needle up as it returns to the original position.
One explanation is that the operator has a steady control of the needle during the slow insertion to reach the consistent target each time. However, there are differences between the experimental setup and what occurs in the operating room for prostate brachytherapy in which high speed hand needle insertion is commonly applied. ${ }^{30}$ The homogeneous phantom in this study versus the heterogeneous skin, muscle, prostate and prostate tumor in brachytherapy is the most significant difference. ${ }^{31}$ Additionally, after insertion into the body, the needle binds to and displaces the prostate and thus deviates from its intended path; known as the splay and roll effect by radiation oncologist, slow insertion speed of the needle to produce small deflection may not be applicable to the clinical procedures because of this effect. ${ }^{32}$

The overall smallest average and standard deviation occurs when using the tight thin grid and the tight thick grid at slow speed. This supports the conclusion that tight grids and slow needle speed reduces the needle deflection.

Improvements on precision and decreased needle deflection can be seen on the $X_{\text {deflection }}$ and $Y_{\text {deflection }}$ data points when the tight thick grid [Fig. 6(d)] was used during slow insertion. Furthermore, at fast insertion speed, which is the approach commonly used in current brachytherapy procedure, the tight thin and tight thick grids produced more data points that were closer to the target $(0,0)$ and within the 2 $\mathrm{mm}$ circle, when compared to the loose thin and loose thick

TABLE V. $p$ values $\left(<0.05\right.$ are bolded) for $X_{\text {deflection }}, Y_{\text {deflection }}$, and $R$ data.

\begin{tabular}{lccc}
\hline \hline & \multicolumn{3}{c}{$p$-value } \\
\cline { 2 - 4 } Factor(s) & $X_{\text {deflection }}$ & $Y_{\text {deflection }}$ & $R$ \\
\hline Tightness & $\mathbf{0 . 0 2 4}$ & $\mathbf{0 . 0 0 0}$ & $\mathbf{0 . 0 0 0}$ \\
Thickness & $\mathbf{0 . 0 0 0}$ & $\mathbf{0 . 0 2 1}$ & 0.297 \\
Speed & 0.247 & $\mathbf{0 . 0 0 0}$ & $\mathbf{0 . 0 0 0}$ \\
Thickness/tightness & $\mathbf{0 . 0 0 0}$ & $\mathbf{0 . 0 0 9}$ & 0.084 \\
Speed/thickness & 0.619 & 0.228 & $\mathbf{0 . 0 1 5}$ \\
Speed/tightness & 0.427 & 0.082 & $\mathbf{0 . 0 3 0}$ \\
Speed/thickness/tightness & 0.834 & 0.720 & 0.115 \\
\hline \hline
\end{tabular}


grids. Overall, the grid tightness (tighter clearance hole) is important to decreasing the needle deflection.

\section{III.C. Deflection significant factors}

\section{III.C.1. Significant factors for $X_{\text {deflection, }} Y_{\text {deflection, }}$ and $R$ deflection data}

ANOVA analysis is used to make simultaneous comparison between two or more means; thus the p-values indicate if there are differences between levels of a factor (main effects) and if differences of one factor depends on the level of other factor(s) (two-way and three-way interactions). ${ }^{27}$ Table $\mathrm{V}$ shows the $p$ value with significant $(p$-value $<0.05)$ marked by bold for the main effect, two-way interaction, and three-way interaction of $X_{\text {deflection }}, Y_{\text {deflection, }}$ and $R$. Significant main effects and twoway interaction included the thickness, tightness, and thickness/tightness for $X_{\text {deflection; }}$; the speed, thickness, tightness, and thickness/tightness for $Y_{\text {deflection; }}$ and the speed, tightness, speed/tightness, and speed/thickness for $R$. One observation that stands out in Table $\mathrm{V}$ is that tightness was significant for all data types $\left(X_{\text {deflection }}, Y_{\text {deflection, }}\right.$, and $R$ ), thus, it is an important factor to consider when decreasing needle deflection. Furthermore, the three-way interaction demonstrated ( $p$ value $>0.05$ ) that the difference observed has only a $5 \%$ probability of not being random, thus performing the assigned condition combination on a certain day did not likely affect the significant outcome of the main effect and two-way interaction.

\section{III.C.2. Multiple comparisons for significant $X_{\text {deflection, }}$ $Y_{\text {deflection, }}$ and $R$ deflection data}

With the thickness/tightness two-way interaction having a significant $p$-value for both the $X_{\text {deflection }}$ and $Y_{\text {deflection }}$ data, along with speed/thickness and speed/tightness for the $R$ data, Tukey's test ${ }^{27}$ was performed to find which averages were significantly different from one another within the twoway interaction. Tukey's test, based on a studentized range distribution $q$, compares all possible pairs of means within the respective two-way interaction. Of the six possible differences in mean for $X_{\text {deflection, }}$, only a subset was calculated to be significant. Similar results hold for the $Y_{\text {deflection }}$ and $R$ data, as shown in Tables VI and VII.

For $X_{\text {deflection }}$ in Table VI, the loose thin grid (Pair II) has significant differences $($ difference $=$ Pair I - Pair II) to the other three grids, as shown by the loose thin grid having a $p$ -

TABLE VI. $X_{\text {deflection }}$ and $Y_{\text {deflection }}$ Tukey's test for significant tightness/thickness two-way interactions.

\begin{tabular}{lllllll}
\hline \hline Data type & \multicolumn{2}{c}{ Pair I } & & \multicolumn{2}{c}{ Pair II } & p-value \\
\cline { 2 - 3 } \cline { 7 - 7 } & Tightness & Thickness & & Tightness & Thickness & \\
\hline$X_{\text {deflection }}$ & Loose & Thick & & Loose & Thin & 0.000 \\
& Tight & Thin & & Loose & Thin & 0.000 \\
& Tight & Thick & & Loose & Thin & 0.000 \\
& & & & & & \\
$Y_{\text {deflection }}$ & Loose & Thick & & Loose & Thin & 0.000 \\
& Tight & Thin & & Loose & Thick & 0.000 \\
& Tight & Thick & & Loose & Thick & 0.000 \\
\hline \hline
\end{tabular}

TABLE VII. $R$ Tukey's test for significant speed/tightness and speed/thickness two-way interactions.

\begin{tabular}{|c|c|c|c|c|c|}
\hline \multirow[t]{2}{*}{ Two-way interaction } & \multicolumn{2}{|c|}{ Pair I } & \multicolumn{2}{|c|}{ Pair II } & \multirow[t]{2}{*}{$p$-value } \\
\hline & Speed & Tightness & Speed & Tightness & \\
\hline \multirow[t]{5}{*}{ Speed/tightness } & Fast & Loose & Slow & Loose & 0.000 \\
\hline & Slow & Tight & Fast & Loose & 0.000 \\
\hline & Fast & Tight & Fast & Loose & 0.000 \\
\hline & Fast & Tight & Slow & Tight & 0.004 \\
\hline & Speed & Thickness & Speed & Thickness & $p$-value \\
\hline \multirow[t]{4}{*}{ Speed/thickness } & Fast & Thin & Slow & Thin & 0.006 \\
\hline & Fast & Thick & Slow & Thin & 0.000 \\
\hline & Slow & Thick & Fast & Thin & 0.000 \\
\hline & Fast & Thick & Slow & Thick & 0.000 \\
\hline
\end{tabular}

value $=0.000(p$-value $<0.05)$ when compared to the other three grids. Furthermore, the loose thin grid had a larger average deflection (for $X_{\text {deflection }}$ data) than the other tightness/thickness combinations (or other grids) in Pair I (see Table IV). Consequently, the loose thin combination was the worst grid to obtain small $X_{\text {deflection. }}$. By similar analysis for $Y_{\text {deflection, }}$, two grids - the loose thin and the loose thick grids (Pair II)-showed significant differences with grids in Pair I, consisting mostly of tight grids; thus, the loose grids should not be used to obtain small $Y_{\text {deflection. }}$.

The results for Tukey's test on the speed/tightness interactions from the $R$ data, shown in Table VII, revealed significant differences mostly between loose grids in Pair II and tight grids in Pair I, with each tightness type present in three out of four comparisons. With regards to the speed/thickness interactions, most of the thin grids (Pair II-thickness), were shown to have a significant difference with most of the thick grids (Pair I-thickness). The average deflection values for $R$, shown in Table IV, demonstrate relative higher deflections in the Pair I group of speed/thickness (mainly fast and thick) compared to the Pair II group (mainly slow and thin). The results strongly support the claim that loose grids and fast speed are the worst conditions to obtain lower $R$.

Collectively, these results again prove the benefits for using the tight grid. Furthermore, significant improvements were made on decreasing the needle deflection during needle insertion as shown by the number of significant $p$-value less than 0.05 when the factor tightness was a main effect or within a two-way interaction.

\section{III.D. Correlation tests}

Before accepting the ANOVA results, the $X_{\text {deflection, }}$ $Y_{\text {deflection, }}$ and $R$ residual data (observation data minus mean) were verified to be independent (plot of residual versus run order of data), normally distributed (normal probability plot of residual), and show homoscedasticity (plot of residuals versus fitted values). ${ }^{27}$ Using MINITAB, abnormal trends did not exist within the data plots. Two correlation tests were performed on the data; the Pearson Correlation test was performed to test deviation magnitudes while the Rayleigh test $^{33}$ detected correlation within the direction of the 
data. Results for the Pearson correlation was 0.086 ( $p$-value $=0.450)$; since the value was insignificant, the magnitude of the $X_{\text {deflection }}$ and $Y_{\text {deflection }}$ data is independent. Rayleigh test produced a value of 0.12 , which was smaller than the critical value of 0.20 ; thus, there was no tendency for a particular $x$ - and $y$ - direction to occur together. Furthermore, correlation did not occur within the data.

\section{CONCLUSIONS}

The grid with tight clearance holes demonstrated to be important and advantageous to decrease needle deflection $\left(X_{\text {deflection, }} Y_{\text {deflection }}, R\right.$ ). Slow needle insertion speed improved the averages and standard deviation of the needle deflection for all conditions within the $Y_{\text {deflection }}$ and $R$ and six out of eight cases within $X_{\text {deflection. Likewise, the best }}$ results for average and standard deviation of needle deflection occurred with a tight grid at slow insertion speed. Although fast hand needle insertion occurs in current brachytherapy procedures, the tight grid improved the averages and standard deviation of the needle deflection when the needle was inserted at fast speed. Furthermore, statistical analysis showed significant $p$-values for $X_{\text {deflection }}, Y_{\text {deflection, }}$ and $R$ when the grid tightness was the main factor.

Results in this study show the potential to improve needle deflection in current brachytherapy procedures when the tight grid is used. Future work includes using the tight grid to perform fast needle insertion experiments using an imaging modality such as ultrasound and computed tomography (CT) on the cadaver or animal model to confirm the benefit of needle placement accuracy within the prostate. Even though slow insertion speed proved to be beneficial in our experimental setup, there are differences between the experimental setup and what occurs clinically in brachytherapy in which high speed hand needle insertion is commonly applied due to the rotation and translation of the prostate. Future work of this research is to investigate the benefit of tight grid for different needle insertion speeds on the cadaver or animal model within the brachytherapy setup.

Additionally, the distance between the grid and phantom can influence needle deflection within the phantom. If the distance the needle travel between the grid and phantom is decreased, smaller needle deflection is possible within the phantom. The effect of distance is a good topic for future study.

\section{ACKNOWLEDGMENTS}

The authors would like to thank Dr. Edward Rothman from the Statistics Department at the University of Michigan for his assistance with the statistical analysis. The authors would also like to acknowledge the support of National Science Foundation Award CMMI\#0825795 and the University of Michigan Radiation Oncology Department.

\section{APPENDIX A: SAMPLE SIZE}

In order to calculate the sample size, ${ }^{27} \mathrm{Eq}$. (A1) for a fixed effects experiment was used. Equation (A1) uses $\Phi$ and the operating characteristic curve $(\alpha=0.05)$ to find the number of replicates to use in the experiment that will be sensitive to the important differences in the conditions. Using a power (probability of rejecting the null hypothesis, with the null hypothesis being the difference between any two conditions mean is $1 \mathrm{~mm}$, when the null is false) of at least 0.85 was used to find the sample size of $n=10$ replicates for this experiment

$$
\Phi^{2}=\frac{n D^{2}}{2 a \sigma^{2}},
$$

where $\Phi^{2}$ is a parameter that related to the noncentrality parameter, $n$ is the number of replicates, $D$ is the difference between any two conditions means, $a$ is the number of levels for the factors, and $\sigma^{2}$ is the variance. A difference between conditions of $D=1 \mathrm{~mm}, a=2$ levels, and a variance $\sigma^{2}=0.49 \mathrm{~mm}^{2}$ were used in Eq. (A1). The variance was obtained from a previous experiment when two operators and three measurement trials were used to obtain the value.

\section{Outliers}

Outliers within each condition combinations were detected using Grubbs' test. ${ }^{28,29}$ Grubbs' test uses the extreme studentized deviate (ESD) method to quantify how far outliers are from the other data points by using the following equation:

$$
Z=\frac{|\mu-v|}{\sigma}
$$

where $Z$ is the ratio used to compare to the critical value, $\mu$ is the mean of the data points within a condition combination, $v$ is the replicate of interest within the condition, and $\sigma$ is the standard deviation of the data points within a condition combination. If $Z$ is bigger than 2.29 (the critical value when the sample size is 10$),{ }^{28,29}$ the value used to calculate $Z$ is an outlier ( $p$-value $<0.05$ ). This method, which uses a twosided t-test, states that the chances are less than $5 \%$ that you encountered an outlier by chance alone, if data were sampled from a Gaussian distribution.

\footnotetext{
a) Author to whom correspondence should be addressed. Electronic mail: cmcgillz@umich.edu

${ }^{1}$ S. Nath, Z. Chen, N. Yue, S. Trumpore, and R. Peshcel, "Dosimetric effects of needle divergence in prostate seed implant using $125 \mathrm{I}$ and 103Pd radioactive seeds," Med. Phys. 27, 1058-1066 (2000).

${ }^{2}$ N. N. Stone and R. G. Stock, "Complications following permanent prostate brachytherapy," Eur. Urol. 41, 427-433 (2002).

${ }^{3}$ J. C. Blasko, T. Mate, J. E. Sylvester, P. D. Grimm, and W. Cavangah, "Brachytherapy for carcinoma of the prostate:Techniques, patient selection, and clinical outcomes," Semin. Radiat. Oncol. 12, 81-94 (2002).

${ }^{4}$ A. T. Porter, J. C. Blasko, P. D. Grimm, S. M. Reddy, and H. Ragde, "Brachytherapy for prostate cancer," Ca-Cancer J. Clin. 45, 165-178 (1995).

${ }^{5}$ S. Jiang, N. Hata, and R. Kikinis, "Needle insertion simulation for imageguided brachytherapy of prostate cancer," 2nd International Conference on Bioinformatics and Biomedical Engineering (Shanghai, China, 2008), pp. 1682-1685.

${ }^{6}$ S. P. DiMaio and S. E. Salcudean, "Needle insertion modeling and simulation,” IEEE Trans. Rob. Autom., 19, 864-875 (2002).

${ }^{7}$ D. Glozman and M. Shoham, "Flexible needle steering and optimal trajectory planning for percutaneous therapies," Medical Image Computing and Computer-Assisted Intervention-MICCAI (Saint-Malo, France, 2004), pp. 137-144.
} 
${ }^{8}$ T. K. Podder, D. P. Clark, J. Sherman, D. Fuller, E. Messing, D. Rubens, J. Strang, R. Brasacchio, L. Liao, W. S. Ng, and Y. Yu, "In vivo motion and force measurement of surgical needle intervention during prostate brachytherapy," Med. Phys. 33, 2915-2922 (2006).

${ }^{9}$ N. Abolhassani, R. V. Patel, and F. Farzam, "Minimization of needle deflection in robot-assisted percutaneous therapy," Int. J. Med. Rob. Comput. Assist. Surg. 3, 140-148 (2007).

${ }^{10}$ H. Kataoka, T. Washio, M. Audette, and K. Mizuhara, "A Model for relations between needle deflection, force, and thickness on needle penetration," Proceedings of the 4th International Conference on Medical Image Computing and Computer-Assisted Intervention, (Utrecht, The Netherlands, 2001), pp. 966-974.

${ }^{11}$ N. Abolhassani, R. Patel, and F. Ayazi, "Effects of different insertion methods on reducing needle deflection," 29th Annual International Conference of the IEEE Engineering in Medicine and Biology Society, (Lyon, France, 2007), pp. 491-494.

${ }^{12}$ H. Kataoka, T. Washio, K. Chinzei, K. Mizuhara, C. Simone, and A. M. Okamura, "Measurement of the tip and friction force acting on a needle during penetration," Medical Image Computing and Computer-Assisted Intervention-MICCAI, (Tokyo, Japan, 2002), pp. 216-223.

${ }^{13}$ A. M. Okamura, C. Simone, and M. D. O'Leary, "Force modeling for needle insertion into soft tissue," IEEE Trans. Biomed. Eng. 51, 1707-1716 (2004).

${ }^{14}$ G. Wan, Z. Wei, L. Gardi, D. B. Downey, and A. Fenster, "Brachytherapy needle deflection evaluation and correction, " Med. Phys. 32, 902-909 (2005)

${ }^{15}$ M. A. Meltsner, N. J. Ferrier, and B. R. Thomadsen, "Observations on rotating needle insertions using a brachytherapy robot," Phys. Med. Biol. 52, 6027-6037 (2007)

${ }^{16}$ T. K. Podder, W. S. Ng, and Y. Yu, "Multi-channel robotic system for prostate brachytherapy," 29th Annual International Conference of the IEEE Engineering in Medicine and Biology Society, (Lyon, France, 2007), pp. 1233-1236.

${ }^{17}$ N. Abolhassani, R. Patel, and M. Moallem, "Needle insertion into soft tissue: A survey," Med. Eng. Phys. 29, 413-431 (2007).

${ }^{18}$ F. W. Mick and K. Zabrouski, U. S. patent 6,579,262 (25 January 2000).

${ }^{19}$ W. F. Whitmore III, W. E. Barzell, and R. F. Wilson, U. S. patent 6,036,632 (28 May 1998)

${ }^{20}$ N. Abolhassani and R. V. Patel, "Deflection of a Flexible Needle during Insertion into Soft Tissue," 28th Annual International Conference of the
IEEE Engineering in Medicine and Biology Society (New York, NY, 2006), pp. 3858-3861.

${ }^{21}$ T. K. Podder, D. P. Clark, J. Sherman, D. Fuller, E. Messing, D. J. Rubens, J. G. Strang, Y. D. Zhang, W. O'Dell, W. S. Ng, and Y. Yu, "Effects of tip geometry of surgical needles: An assessment of force and deflection," Third European Medical and Biological Engineering Conference (Prague, Czech Republic, 2005).

${ }^{22}$ Y. Yu, T. K. Podder, Y. Zhang, W. S. Ng, V. Misic, J. Sherman, L. Fu D. Fuller, E. Messing, D. Rubens, J. Strang, and R. Brasacchio, "RobotAssisted Prostate Brachytherapy" Medical Image Computing and ComputerAssisted Intervention-MICCAI (Copenhagen, Denmark, 2006), pp. 41-49.

${ }^{23}$ Measurement Systems Analysis (Daimler Chrysler, Ford Motor, and General Motors, 2002)

${ }^{24}$ D. H. Stamatis, Six Sigma and Beyond: Statistical Process Control (St. Lucie Press, Boca Raton, Florida, 2002).

${ }^{25}$ S. G. Shina, Six Sigma Electronics Design and Manufacturing (McGrawHill, New York, 2002).

${ }^{26}$ T. K. Podder, D. P. Clark, D. Fuller, J. Sherman, W. S. Ng, L. Liao D. Rubens, J. Strang, E. Messing, Y. D. Zhang, and Y. Yu, "Effects of velocity modulation during surgical needle insertion," 27th Annual International Conference of the IEEE Engineering in Medicine and Biology Society, (Shanghai, China, 2005), pp. 5766-5770.

${ }^{27}$ D. C. Montgomery, Design and Analysis of Experiments (Wiley, New York, 2005).

${ }^{28}$ V. Barnett and T. Lewis, Outliers in Statistical Data (John Wiley \& Sons, New York, 1994).

${ }^{29}$ B. Iglewicz and D. C. Hoaglin, How to Detect and Handle Outliers (American Society for Quality Control, Wisconsin, 1993).

${ }^{30}$ C. S. McGill, J. Schwartz, J. Z. Moore, P. W. McLaughlin, and A. J. Shih, "Accurate insertion of stiffer needle using fast speed device for brachytherapy," (submitted).

${ }^{31}$ R. Alterovitz, K. Goldberg, J. Pouliot, R. Taschereau, and I-C. Hsu, "Needle insertion and radioactive seed implantation in human tissues: Simulation and sensitivity analysis," IEEE Int. Conf. Rob. Autom., 2 1793-1799 (2003)

${ }^{32}$ P. L. Roberson, V. Narayana, D. L. McShan, R. J. Winfield, and P. W. McLaughlin, "Source placement error for permanent implant of the prostate," Med. Phys. 24, 251-257 (1997).

${ }^{33}$ K. V. Mardia, Statistics of Directional Data (Academic, New York, 1972). 